\title{
Association between Breastfeeding and Prevalence of Diabetes in Korean Parous Women: The Korea National Health and Nutrition Examination Survey, 2010-2014
}

\author{
Ha-Nui Kim, Young-Ah Jung, Li-Ly Kang, Hoon-Ki Park*, Hwan-Sik Hwang, Kye-Yeung Park \\ Department of Family Medicine, Hanyang University College of Medicine, Seoul, Korea
}

See editorial commentary page on 271

Background: It is well known that breastfeeding has a significant impact on the health of mothers and children. With the growing importance of breastfeeding, the present study aimed to investigate the relationship between breastfeeding and the prevalence of diabetes in Korean parous women.

Methods: The data of 5,448 premenopausal parous women aged 20-49 years who agreed to participate in the 5th6th Korea National Health and Nutrition Examination Survey were analyzed in this study. Control group included women who had not breastfed. The subjects who had breastfed were classified into three groups based on the duration of breastfeeding: 0-6 months, 6-12 months, and >12 months. The variables included age, body mass index, education level, income, alcohol drinking, smoking, family history of diabetes, use of oral contraceptives, the number of pregnancies, and regular exercise.

Results: Among the subjects, the prevalence of diabetes was significantly lower in women who had breastfed compared to those who had not, with an odds ratio of 0.534 (95\% confidence interval [CI], 0.289-0.976) in women who breastfed for 0-6 months and 0.575 (95\% CI, 0.321-0.990) in women who breastfed for 6-12 months (both $\mathrm{P}<0.05$ ). Conclusion: The present study found a reduced prevalence of diabetes in women who had breastfed compared to those who had not. However, no association between the duration of breastfeeding and the prevalence of diabetes could be found.

Keywords: Breast Feeding; Diabetes Mellitus; Parity; Prevalence

Received: February 24, 2017, Revised: August 24, 2017, Accepted: September 4, 2017

*Corresponding Author: Hoon-Ki Park https://orcid.org/0000-0002-8242-0943

Tel: +82-2-2290-8740, Fax: +82-2-2281-7279, E-mail: hoonkp@hanyang.ac.kr 


\section{INTRODUCTION}

World Breastfeeding Week is celebrated annually from 1 to 7 August to encourage breastfeeding and improve the health of babies worldwide. The World Health Organization and the United Nations International Children's Emergency Fund recommendations on breastfeeding are as follows: initiation of breastfeeding within the first hour after the birth; exclusive breastfeeding for the first 6 months; and continued breastfeeding for 2 years or more, together with safe, nutritionally adequate, age appropriate, responsive complementary feeding starting in the 6th month. ${ }^{1-3)}$

It is well known that breastfeeding has a significant impact on the health of mothers and children. Breastfed children show improvement in cognitive development, emotional stability, and social integration in the course of their growth. ${ }^{4)}$ Breastfeeding also prevents the development of allergic rhinitis and asthma even in children with a family history of atopic dermatitis. ${ }^{5,6)}$ Furthermore, breastfeeding seems to have a small, but consistent protective effect against obesity in children; ${ }^{7}$ moreover, it has been associated with a lower prevalence of hypertension and hypercholesterolemia in adulthood. In addition, breastfeeding mothers benefit from earlier return to prepregnancy weight. Fur- thermore, breastfeeding decreases the risk of breast and ovarian cancer in premenopausal women, and that of hip fracture and osteoporosis in postmenopausal women., ${ }^{8,9)}$

While the importance of breastfeeding is becoming clear, there have been many studies on the association between breastfeeding and diabetes in other countries, including the United States, and the results of previous studies have shown that women who had never breastfed or never exclusively breastfed had an increased risk of type 2 diabetes. ${ }^{10-14)}$ Failure to breastfeed was also found associated with other cardiovascular risk factors, such as myocardial infarction and the metabolic syndrome. ${ }^{15)}$ However, few studies were performed on Korean women in this regard. Therefore, this study was conducted to evaluate the relationship between breastfeeding and the prevalence of diabetes mellitus in Korean young women aged 20-49 years (younger than Korean average menopause age). Few studies on women who are younger than menopausal age are currently available. This study aimed to investigate the association between breastfeeding and the prevalence of diabetes mellitus in Korean parous women by using the data from the Korea National Health and Nutrition Examination Survey (KHANES).

Table 1. Baseline characteristics of study subjects based on the duration of breastfeeding

\begin{tabular}{|c|c|c|c|c|c|}
\hline \multirow{2}{*}{ Characteristic } & \multicolumn{4}{|c|}{ Duration of breastfeeding (mo) } & \multirow{2}{*}{ P-value } \\
\hline & None $(\mathrm{N}=1,181)$ & $0-6(\mathrm{~N}=1,240)$ & $6-12(N=927)$ & $>12(\mathrm{~N}=2,100)$ & \\
\hline Diabetes & $5.1(0.8)$ & $2.2(0.5)$ & $2.6(0.6)$ & $3.2(0.5)$ & $0.021^{*}$ \\
\hline $\operatorname{Age}(y)$ & $40.76 \pm 0.2$ & $38.45 \pm 0.2$ & $38.57 \pm 0.3$ & $39.06 \pm 0.1$ & $0.078^{\dagger}$ \\
\hline Body mass index $\left(\mathrm{kg} / \mathrm{m}^{2}\right)$ & $23.17 \pm 0.1$ & $22.65 \pm 0.1$ & $23.00 \pm 0.1^{\ddagger}$ & $23.31 \pm 0.1^{\ddagger}$ & $0.003^{\dagger}$ \\
\hline \multicolumn{6}{|l|}{ Smoking status } \\
\hline Non-smoker & $85.1(1.3)$ & $84.3(1.3)$ & $82.6(1.6)$ & $87.5(0.9)$ & $0.020^{*}$ \\
\hline Ex-smoker & $6.2(0.8)$ & $8.3(0.9)$ & $11.1(1.3)$ & $7.7(0.7)$ & \\
\hline Current-smoker & $8.7(1.0)$ & $7.5(0.9)$ & $6.3(1.1)$ & $4.8(0.6)$ & \\
\hline \multicolumn{6}{|l|}{ Alcohol drinking } \\
\hline None & $7.7(0.9)$ & $7.1(0.8)$ & $9.0(1.1)$ & $7.8(0.7)$ & $0.020^{*}$ \\
\hline Mild to moderate & $85.3(1.2)$ & $85.8(1.2)$ & $85.6(1.4)$ & $86.1(0.9)$ & \\
\hline Severe & $7.0(0.9)$ & $7.1(0.9)$ & $5.5(0.9)$ & $6.0(0.6)$ & \\
\hline Family history of diabetes & $26.8(1.6)$ & $23.4(1.5)$ & $22.7(1.6)$ & $22.7(1.1)$ & $0.021^{*}$ \\
\hline Use of oral contraceptive & $9.6(1.1)$ & $12.5(1.1)$ & $9.8(1.1)$ & $8.6(0.7)$ & $0.020^{*}$ \\
\hline Regular exercise & $29.6(1.6)$ & $33.8(1.7)$ & $35.3(1.8)$ & $36.6(1.2)$ & $0.020^{*}$ \\
\hline \multicolumn{6}{|l|}{ No. of pregnancies } \\
\hline 1,2 & $42.6(1.7)$ & $52.0(1.7)$ & $50.7(1.9)$ & $39.0(1.2)$ & $0.020^{*}$ \\
\hline 3,4 & $43.8(1.7)$ & $37.9(1.6)$ & $38.4(1.9)$ & $44.9(1.3)$ & \\
\hline$\geq 5$ & $13.8(1.2)$ & $10.2(1.0)$ & $10.9(1.3)$ & $16.0(1.0)$ & \\
\hline \multicolumn{6}{|l|}{ Income } \\
\hline Low & $8.1(1.0)$ & $6.1(0.9)$ & $8.5(1.1)$ & $6.9(0.9)$ & $0.020^{*}$ \\
\hline Middle & $58.5(1.8)$ & $61.9(1.7)$ & $65.8(1.8)$ & $66.1(1.3)$ & \\
\hline High & $33.4(1.7)$ & $31.9(1.6)$ & $25.7(1.7)$ & $26.9(1.2)$ & \\
\hline \multicolumn{6}{|l|}{ Education level } \\
\hline Elementary & $3.8(0.7)$ & $2.1(0.5)$ & $2.0(0.6)$ & $3.9(0.6)$ & $0.020^{*}$ \\
\hline Middle school & $6.0(0.9)$ & $4.4(0.8)$ & $4.9(0.9)$ & $7.5(0.7)$ & \\
\hline High school & $54.4(1.8)$ & $44.7(1.7)$ & $50.8(2.0)$ & $45.6(1.3)$ & \\
\hline College or higher & $35.8(1.7)$ & $48.8(1.8)$ & $42.3(2.0)$ & $43.0(1.3)$ & \\
\hline
\end{tabular}

Values are presented as mean \pm standard error or \% (standard error).

${ }^{*}$ Categorical variables were analyzed using the complex sampling chi-square test. ${ }^{\dagger}$ Continuous variables were analyzed using complex sampling analysis of variance. ${ }^{\mathrm{t} P}<0.05$ by post hoc analyses when compared with subjects who did not breastfeed. 


\section{METHODS}

The KHANES was conducted to assess Korean health and nutritional status by extracting a nationwide representative probability sample from households representing the entire population. The present study was based on the data from the 5th-6th KHANES (2010-2014), which was a cross-sectional national survey, including comprehensive information on national health levels and behaviors. Of the 41,102 participants, 22,456 women were included. Of 6,486 women aged between 20 and 49 years, 976 nulliparous and 62 menopausal women were excluded. Finally, the data of 5,448 pre-menopausal parous women aged $20-49$ years were analyzed in the present study. The parous women were defined as women who had experience of delivery, including normal or preterm delivery, regardless of live birth.

Patients with diabetes were defined as those having a fasting glucose level $\geq 7 \mathrm{mmol} / \mathrm{L}$, having been diagnosed by a physician as having diabetes, or using antidiabetic medications or injected insulin. Each participant reported whether she had ever been diagnosed by a physician as having diabetes. In addition, the subjects were classified according to whether they had breastfeeding experience. The subjects were asked to respond to the following questions: "Have you ever breastfed?"; "How many children have you breastfed?"; and "How long have you been breastfeeding?" Those who had breastfed were classified into three groups based on the total duration of breastfeeding regardless of the number of children: 0-6 months, 6-12 months, and >12 months. The covariables included age, body mass index (BMI), education level, income, alcohol drinking, smoking, family history of diabetes mellitus, use of oral contraceptives, the number of pregnancies, and regular exercise.

Information was collected during the health interview survey. The BMI was a measure of body fat based on height and weight, and was expressed in $\mathrm{kg} / \mathrm{m}^{2}$. Education level was categorized into four groups by the subject's highest achieved level: below elementary school, middle school, high school, and college or higher. Income was also divided equally into three groups based on the monthly income of the entire household: low, middle, and high. Smoking status was divided into three categories: nonsmoker, ex-smoker, and current smoker. Alcohol drinking was divided into three groups: group "none" included participants who had never been drinking alcohol. Severe drinking was defined as drinking $\geq 5$ drinks each time at least 2 or more times a week. Other participants were classified as mild to moderate drinking. Regular exercise was defined as $\geq 30$ minutes of walking and $\geq 5$ days a week. A positive family history of diabetes was considered when either mother or father of participant had diabetes mellitus. Menopause status was indicated as 'yes' for participants who answered natural or artificial menopause.

For the subjects in the 2010-2014 KHANES to represent the entire Korean population, we applied weight sampling to take the complex sampling method into account. Mean values of continuous variables such as age and BMI were compared among the four groups using analysis of variance followed by post hoc analyses. The chi-square test was used to analyze the distribution of each factor across the duration of breastfeeding. After adjusting for all variables, including age, BMI, education level, income, alcohol drinking, smoking, family history of diabetes, use of oral contraceptives, the number of pregnancies, and regular exercise, complex sampling logistic regression analysis was performed to determine the association between breastfeeding and the prevalence of diabetes mellitus. Statistical analysis was performed using IBM SPSS ver. 20.0 for Windows (IBM Corp., Armonk, NY, USA), and a P-value $<0.05$ was considered statistically significant. This nationwide survey was approved by the Institutional Review Board of the Korea Centers for Disease Control and Prevention (IRB approval no., 2007-02-CON-04-P).

Table 2. Odd ratios (95\% confidence intervals) of having diabetes after adjustment for multiple covariables

\begin{tabular}{|c|c|c|}
\hline Variable & Unadjusted & Adjusted $^{*}$ \\
\hline Age & $1.090(1.054-1.128)$ & $1.064(1.024-1.107)$ \\
\hline BMl & $1.258(1.214-1.304)$ & 1.255 (1.205-1.308) \\
\hline \multicolumn{3}{|l|}{ Breastfeeding } \\
\hline Never & 1 & 1 \\
\hline Ever & $0.538(0.360-0.803)$ & $0.590(0.383-0.909)$ \\
\hline \multicolumn{3}{|l|}{ Alcohol drinking } \\
\hline No & 1 & 1 \\
\hline Mild to moderate & $0.409(0.233-0.717)$ & $0.426(0.241-0.751)$ \\
\hline Severe & $0.523(0.204-1.338)$ & $0.513(0.183-1.442)$ \\
\hline \multicolumn{3}{|l|}{ Smoking } \\
\hline Nonsmoker & 1 & 1 \\
\hline Ex-smoker & $0.741(0.364-1.507)$ & $0.879(0.420-1.841)$ \\
\hline Current smoker & $0.590(0.271-1.283)$ & $0.401(0.160-1.002)$ \\
\hline \multicolumn{3}{|c|}{ Family history of diabetes } \\
\hline No & 1 & 1 \\
\hline Yes & $2.892(1.980-4.224)$ & 2.991 (1.978-4.525) \\
\hline \multicolumn{3}{|l|}{ No. of pregnancies } \\
\hline 1,2 & 1 & 1 \\
\hline 3,4 & $1.155(0.762-1.752)$ & $0.908(0.576-1.432)$ \\
\hline$\geq 5$ & $2.347(1.461-3.770)$ & $1.528(0.903-2.585)$ \\
\hline \multicolumn{3}{|l|}{ Use of $O C$} \\
\hline No & 1 & 1 \\
\hline Yes & $1.126(0.587-2.161)$ & $1.030(0.527-2.014)$ \\
\hline \multicolumn{3}{|l|}{ Regular exercise } \\
\hline No & 1 & 1 \\
\hline Yes & $0.836(0.559-1.251)$ & $0.772(0.496-1.200)$ \\
\hline \multicolumn{3}{|l|}{ Income } \\
\hline Low & 1 & 1 \\
\hline Middle & $0.503(0.281-0.903)$ & $0.520(0.264-1.023)$ \\
\hline High & $0.476(0.255-0.888)$ & $0.605(0.285-1.282)$ \\
\hline \multicolumn{3}{|l|}{ Education level } \\
\hline Elementary & 1 & 1 \\
\hline Middle school & $0.829(0.304-2.259)$ & $1.643(0.532-5.074)$ \\
\hline High school & $0.600(0.255-1.416)$ & $1.560(0.604-4.028)$ \\
\hline College or higher & $0.258(0.104-0.642)$ & $0.927(0.333-2.579)$ \\
\hline
\end{tabular}

Values are presented as odds ratio (95\% confidence interval).

BMI, body mass index; OC, oral contraceptive.

*Complex sample logistic regression analyses were adjusted for age, BMl, alcohol drinking, smoking, family history of diabetes, menopause, number of pregnancies, use of $\mathrm{OC}$, income, education level, and regular exercise. 


\section{RESULTS}

Of the 5,448 subjects, 1,181 parous women (21.7\%) had never breastfed and 4,267 parous women had breastfed. According to the duration of breastfeeding, $22.8 \%$ of women breastfed for 0-6 months, $17.0 \%$ of women breastfed for 6-12 months, and 38.5\% of women breastfed for $>12$ months. The prevalence of diabetes among breastfeeding women was $2.2 \%, 2.6 \%, 3.2 \%$ in women who breastfed for 0-6 months, 6-12 months, and $>12$ months, respectively. The baseline characteristics of the subjects based on the duration of breastfeeding are shown in Table 1. Participants who had not breastfed showed higher prevalence of diabetes mellitus than participants who had breastfed. BMI, smoking, alcohol drinking, family history of diabetes, use of oral contraceptives, the number of pregnancies, education level, regular exercise, and income were significantly different according to the duration of breastfeeding (Table 1).

The factors associated with diabetes mellitus, odd ratio (OR), and 95\% confidence intervals (CI) are shown in Table 2. Logistic regression analysis with the presence of diabetes as dependent variable showed that OR of diabetes prevalence was 0.538 (95\% CI, 0.360-0.803) for those who breastfed compared with those who did not. After adjusting for all variables, including age, BMI, education level, income, alcohol drinking, smoking, family history of diabetes, number of pregnancies, use of oral contraceptive, and regular exercise, the OR of diabetes mellitus presence was 0.590 (95\% CI, 0.383-0.909) for those who breastfed compared with those who did not, showing a statistically significant association $(\mathrm{P}<0.05)$ (Table 2).

The ORs and 95\% CI for diabetes according to the duration of breastfeeding are shown in Figure 1. The results of the analysis showed that without adjusting for variables, the ORs of diabetes were 0.420 (95\% CI, 0.237-0.741), 0.504 (95\% CI, 0.287-0.885), and 0.622 (95\% CI, 0.394-0.982) for women who breastfed for 0-6 months, 6-12 months, and $>12$ months, respectively, compared to those who did not, showing a statistically significant difference. After adjusting for all variables, including age, BMI, education level, income, alcohol drinking, smoking, family history of diabetes, number of pregnancies, use of oral contraceptives, and regular exercise, the ORs of diabetes were 0.534 (95\% CI, 0.289-0.976), and 0.575 (95\% CI, 0.321-0.990) for women who breastfed for 0-6 months and 6-12 months, respectively, compared to those who did not, showing a statistically significant association $(\mathrm{P}<0.05)$ (Figure 1).

\section{DISCUSSION}

In this cross-sectional study of 5,448 premenopausal parous women, we found that breastfeeding was associated with diabetes mellitus. The results showed that the prevalence of diabetes mellitus was reduced in women who breastfed compared with those who did not. In addition, we found that the prevalence of diabetes was lower in women who breastfed for 0-6 months and 6-12 months compared with those who did not.

Many previous studies assessed the association between breastfeeding and diabetes mellitus. Zhang et al. ${ }^{10)}$ found that women who did not breastfeed had an increased risk of diabetes mellitus compared with those who did (OR, 1.30; 95\% CI, 1.11-1.53), and that the risk was reduced in women with a duration of breastfeeding of 0-6 months (OR, 0.81; 95\% CI, 0.67-0.98) and 6-12 months (OR, 0.46; 95\% CI, 0.260.84). In a study by Stuebe et al., ${ }^{11)}$ which was a cohort study performed on women in the United States, a longer duration of breastfeeding was associated with a lower prevalence of type 2 diabetes mellitus in women. Villegas et al. ${ }^{12)}$ also found that women who had breastfed had a lower risk of diabetes mellitus than those who had never breastfed (relative risk, 0.88; 95\% CI, 0.76-1.02; $\mathrm{P}=0.08$ ). A longer duration of breastfeeding was found associated with a reduced risk of type 2 diabetes mellitus. Liu et al. ${ }^{14)}$ reported a $14 \%$ reduced risk of diabetes mellitus in women who breastfeed compared with those who do not. The present study also found that the prevalence of diabetes mellitus was reduced by about $50 \%$ in those who breastfed for 0-6 months and 6-12
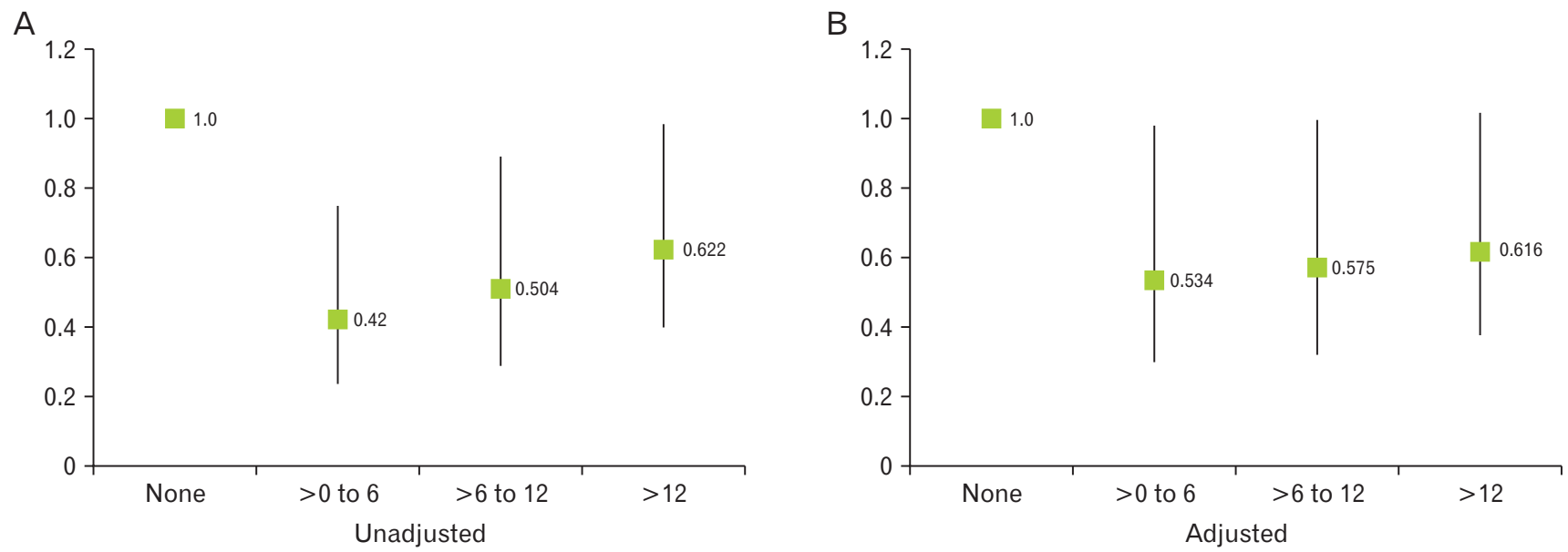

Figure 1. (A, B) Odd ratios for diabetes according to the duration of breastfeeding. Complex sample logistic regression analyses were adjusted for age, body mass index, alcohol drinking, smoking, family history of diabetes, number of pregnancies, use of oral contraceptive, income, education level, and regular exercise. 
months compared to those who did not breastfeed. Compared to other studies mentioned above, this study showed very low ORs. This study included women who were aged 20-49 years. However, other studies included older women in the age group of 50-80 years. Moreover, this study had a relatively low prevalence of diabetes mellitus. As women aged below 50 years are less exposed to environmental factors, breastfeeding could be more affective to them. These factors might have influence the lower ORs of diabetes mellitus compared to other studies.

In this study, we found that the prevalence of diabetes mellitus was reduced in women who had breastfed compared to those who did not. However, the association between the duration of breastfeeding and the prevalence of diabetes mellitus could not be found. More specific and continuous studies related to this relationship should be conducted in the future.

The group of women who breastfed $>12$ months showed no statistically significant association with diabetes mellitus. There was no evidence that longer duration of breastfeeding was related to an increased amount of breastfeeding. Every woman had different style of breastfeeding related to frequency, time of breastfeeding, etc. In this study, information was collected about the total duration of breastfeeding in lifetime, not about the way of breastfeeding. We consider that as the duration of breastfeeding increases, the difference between the duration of breastfeeding and a real amount of breastfeeding also increases. This could be the reason why the group of women who breastfed $>12$ months showed no statistically significant association with diabetes mellitus. In addition, unnecessarily long duration of breastfeeding might have no association with the real prevalence of diabetes mellitus. Many studies assed the effect of long-term breastfeeding that lasted $<12$ months. ${ }^{10,11,13,14)}$ However, the effect of breastfeeding for more than 12 months was rarely evaluated.

The mechanisms underlying the association between breastfeeding and diabetes mellitus had not yet been clearly elucidated. However, several studies had shown that breastfeeding improved insulin sensitivity and glucose intolerance, which might reduce the risk of diabetes or delay its development. It was also reported that breastfeeding was associated with improved fasting glucose and reduced insulin levels, especially in those with recent gestational diabetes mellitus (GDM). ${ }^{16}$ ) Breastfeeding had been reported to improve pancreatic beta-cell function, ${ }^{17)}$ and to have beneficial effects on insulin sensitivity and glucose and lipid metabolism in women with GDM. ${ }^{18,19)}$ Furthermore, it was reported in an animal study that breastfeeding increased insulin sensitivity and reduced plasma glucose concentration. ${ }^{20)}$ Blood glucose and insulin levels in lactating rats decreased by $20 \%$ and $35 \%$, respectively, when compared with the levels in non-lactating rats. ${ }^{21)}$ The long-term effects of breastfeeding were to influence pituitary hormone levels ${ }^{22,23)}$ and to affect the hypothalamic-pituitary axis. ${ }^{24,25)}$

This study had several limitations. First, the cross-sectional design of this study prevented identification of a causal relationship between the prevalence of diabetes mellitus and breastfeeding. Second, recall bias might have existed, because the duration of breastfeeding was mea- sured using self-directed questionnaires. Third, as the women with a history of GDM had an increased hazard for developing diabetes mellitus, ${ }^{26)}$ it should be included as a covariable. However, we failed to add it because of the lack of data.

With growing emphasis on its importance, people are increasingly concerned about breastfeeding. Although the rate of breastfeeding in South Korea was low in 2000, it has been increasing gradually over the past 10 years. ${ }^{27)}$ However, continuous efforts are needed to further increase the rate of breastfeeding in the future.

In conclusion, the present study found that the risk of diabetes mellitus was reduced in women who breastfed when compared with those who did not. Although various studies had been conducted in this regard, the precise mechanism for this relationship has not yet been elucidated. Further studies should be performed considering each duration of lactation as a confounder for prevalence of diabetes mellitus.

\section{CONFLICT OF INTEREST}

No potential conflict of interest relevant to this article was reported.

\section{REFERENCES}

1. World Health Organization. Health topics: breastfeeding [Internet]. Geneva: World Health Organization [cited 2016 Dec 20]. Available from: http://www.who.int/topics/breastfeeding/en.

2. World Health Organization. Health topics: nutrition topics: exclusive breastfeeding [Internet]. Geneva: World Health Organization [cited 2016 Dec 20]. Available from: http://www.who.int/nutrition/topics/ exclusive_breastfeeding/en.

3. United Nations Children's Fund. Nutrition: breastfeeding: recommendations for optimal breastfeeding [Internet]. New York (NY): United Nations International Children's Emergency Fund [cited 2016 Dec 20]. Available from: https://www.unicef.org/nutrition/index_24824.html.

4. Kramer MS, Aboud F, Mironova E, Vanilovich I, Platt RW, Matush L, et al. Breastfeeding and child cognitive development: new evidence from a large randomized trial. Arch Gen Psychiatry 2008;65:578-84.

5. Mimouni Bloch A, Mimouni D, Mimouni M, Gdalevich M. Does breastfeeding protect against allergic rhinitis during childhood?: a meta-analysis of prospective studies. Acta Paediatr 2002;91:275-9.

6. Gdalevich M, Mimouni D, Mimouni M. Breast-feeding and the risk of bronchial asthma in childhood: a systematic review with meta-analysis of prospective studies. J Pediatr 2001;139:261-6.

7. Arenz S, Ruckerl R, Koletzko B, von Kries R. Breast-feeding and childhood obesity: a systematic review. Int J Obes Relat Metab Disord 2004; 28:1247-56.

8. Turck D. Breast feeding: health benefits for child and mother. Arch Pediatr 2005;12 Suppl 3:S145-65.

9. Lee SY, Kim MT, Kim SW, Song MS, Yoon SJ. Effect of lifetime lactation on breast cancer risk: a Korean women's cohort study. Int J Cancer 2003;105:390-3.

10. Zhang BZ, Zhang HY, Liu HH, Li HJ, Wang JS. Breastfeeding and maternal hypertension and diabetes: a population-based cross-sectional study. Breastfeed Med 2015;10:163-7. 
11. Stuebe AM, Rich-Edwards JW, Willett WC, Manson JE, Michels KB. Duration of lactation and incidence of type 2 diabetes. JAMA 2005;294:2601-10.

12. Villegas R, Gao YT, Yang G, Li HL, Elasy T, Zheng W, et al. Duration of breast-feeding and the incidence of type 2 diabetes mellitus in the Shanghai Women's Health Study. Diabetologia 2008;51:258-66.

13. Schwarz EB, Brown JS, Creasman JM, Stuebe A, McClure CK, van den Eeden SK, et al. Lactation and maternal risk of type 2 diabetes: a population-based study. Am J Med 2010;123:863.

14. Liu B, Jorm L, Banks E. Parity, breastfeeding, and the subsequent risk of maternal type 2 diabetes. Diabetes Care 2010;33:1239-41.

15. Stuebe A. The risks of not breastfeeding for mothers and infants. Rev Obstet Gynecol 2009;2:222-31.

16. Gunderson EP, Hedderson MM, Chiang V, Crites Y, Walton D, Azevedo RA, et al. Lactation intensity and postpartum maternal glucose tolerance and insulin resistance in women with recent GDM: the SWIFT cohort. Diabetes Care 2012;35:50-6.

17. McManus RM, Cunningham I, Watson A, Harker L, Finegood DT. Beta-cell function and visceral fat in lactating women with a history of gestational diabetes. Metabolism 2001;50:715-9.

18. Much D, Beyerlein A, Roßbauer M, Hummel S, Ziegler AG. Beneficial effects of breastfeeding in women with gestational diabetes mellitus. Mol Metab 2014;3:284-92.

19. Kjos SL, Henry O, Lee RM, Buchanan TA, Mishell DR Jr. The effect of lactation on glucose and lipid metabolism in women with recent gestational diabetes. Obstet Gynecol 1993;82:451-5.
20. Jones RG, Ilic V, Williamson DH. Physiological significance of altered insulin metabolism in the conscious rat during lactation. Biochem J 1984;220:455-60.

21. Burnol AF, Leturque A, Ferre P, Kande J, Girard J. Increased insulin sensitivity and responsiveness during lactation in rats. Am J Physiol 1986;251(5 Pt 1):E537-41.

22. Amico JA, Johnston JM, Vagnucci AH. Suckling-induced attenuation of plasma cortisol concentrations in postpartum lactating women. Endocr Res 1994;20:79-87.

23. De Zegher F, Spitz B, van den Berghe G, Lemmens D, Vanweser K, Keppens $\mathrm{K}$, et al. Postpartum hyperprolactinemia and hyporesponsiveness of growth hormone (GH) to GH-releasing peptide. J Clin Endocrinol Metab 1998;83:103-6.

24. Lankarani-Fard A, Kritz-Silverstein D, Barrett-Connor E, GoodmanGruen D. Cumulative duration of breast-feeding influences cortisol levels in postmenopausal women. J Womens Health Gend Based Med 2001;10:681-7.

25. Groer MW, Davis MW, Hemphill J. Postpartum stress: current concepts and the possible protective role of breastfeeding. J Obstet Gynecol Neonatal Nurs 2002;31:411-7.

26. Ratner RE. Prevention of type 2 diabetes in women with previous gestational diabetes. Diabetes Care 2007;30 Suppl 2:S242-5.

27. Chung SH, Kim HR, Choi YS, Bae CW. Trends of breastfeeding rate in Korea (1994-2012): comparison with OECD and other countries. J Korean Med Sci 2013;28:1573-80. 